\title{
A case-matched study of stereotactic radiosurgery for patients with multiple brain metastases: comparing treatment results for $1-4$ vs $\geq 5$ tumors
}

\author{
Clinical article
}

\author{
Masaaki Yamamoto, M.D., ${ }^{1,2}$ Takuya Kawabe, M.D., ${ }^{1,3}$ Yasunori Sato, Ph.D., ${ }^{4}$ \\ Yoshinori Higuchi, M.D., ${ }^{5}$ TAdashi Nariai, M.D., ${ }^{6}$ Bierta E. Barfod, M.D., ${ }^{1}$ \\ Hidetoshi Kasuya, M.D., ${ }^{2}$ and Yoichi Urakawa, M.D. ${ }^{1}$
}

\begin{abstract}
${ }^{1}$ Katsuta Hospital Mito GammaHouse, Ibaraki; ${ }^{2}$ Department of Neurosurgery, Tokyo Women's Medical University Medical Center East, Tokyo; ${ }^{3}$ Department of Neurosurgery, Kyoto Prefectural University of Medicine Graduate School of Medical Sciences, Kyoto; ${ }^{4}$ Clinical Research Center, Chiba University Graduate School of Medicine, Chiba; ${ }^{5}$ Department of Neurosurgery, Chiba University Graduate School of Medicine, Chiba; and ${ }^{6}$ Department of Neurosurgery, Graduate School, Tokyo Medical and Dental University School of Medicine, Tokyo, Japan
\end{abstract}

\begin{abstract}
Object. Although stereotactic radiosurgery (SRS) alone for patients with 4-5 or more tumors is not a standard treatment, a trend for patients with 5 or more tumors to undergo SRS alone is already apparent. The authors' aim in the present study was to reappraise whether SRS results for $\geq 5$ tumors differ from those for 1-4 tumors.

Methods. This institutional review board-approved retrospective cohort study used the authors' database of prospectively accumulated data that included 2553 consecutive patients who underwent SRS, not in combination with concurrent whole-brain radiotherapy, for brain metastases (METs) between 1998 and 2011. These 2553 patients were divided into 2 groups: 1553 with tumor numbers of 1-4 (Group A) and 1000 with $\geq 5$ tumors (Group B). Because there was considerable bias in pre-SRS clinical factors between Groups A and B, a case-matched study was conducted. Ultimately, 1096 patients (548 each in Groups A and B) were selected. The standard Kaplan-Meier method was used to determine post-SRS survival and the post-SRS neurological death-free survival times. Competing risk analysis was applied to estimate cumulative incidences of local recurrence, repeat SRS for new lesions, neurological deterioration, and SRS-induced complications.

Results. The post-SRS median survival time was significantly longer in the 548 Group A patients (7.9 months, 95\% CI 7.0-8.9 months) than in the 548 Group B patients (7.0 months 95\% [CI 6.2-7.8 months], HR 1.176 [95\% CI $1.039-1.331], \mathrm{p}=0.01)$. However, incidences of neurological death were very similar: $10.6 \%$ in Group A and $8.2 \%$ in Group B $(\mathrm{p}=0.21)$. There was no significant difference between the groups in neurological death-free survival intervals (HR $0.945,95 \%$ CI $0.636-1.394, \mathrm{p}=0.77)$. Furthermore, competing risk analyses showed that there were no significant differences between the groups in cumulative incidences of local recurrence (HR $0.577,95 \%$ CI $0.312-1.069, \mathrm{p}=0.08)$, repeat SRS (HR 1.133, 95\% CI 0.910-1.409, $\mathrm{p}=0.26)$, neurological deterioration $(\mathrm{HR} 1.868$, 95\% CI 0.608-1.240, $\mathrm{p}=0.44$ ), and major SRS-related complications (HR 1.105, 95\% CI 0.490-2.496, p = 0.81).

In the authors' cohort, age $\leq 65$ years, female sex, a Karnofsky Performance Scale score $\geq 80 \%$, cumulative tumor volume $\leq 10 \mathrm{~cm}^{3}$, controlled primary cancer, no extracerebral METs, and neurologically asymptomatic status were significant factors favoring longer survival equally in both groups.

Conclusions. This retrospective study suggests that increased tumor number is an unfavorable factor for longer survival. However, the post-SRS median survival time difference, 0.9 months, between the two groups is not clinically meaningful. Furthermore, patients with 5 or more METs have noninferior results compared to patients with 1-4 tumors, in terms of neurological death, local recurrence, repeat SRS, maintenance of good neurological state, and SRS-related complications. A randomized controlled trial should be conducted to test this hypothesis. (http://thejns.org/doi/abs/10.3171/2013.3.JNS121900)
\end{abstract}

\section{KeY Words • brain metastases • radiation therapy • stereotactic radiosurgery - Gamma Knife • tumor number • oncology}

\footnotetext{
Abbreviations used in this paper: KPS = Karnofsky Performance Scale; MET = metastasis; MST = median survival time; RPA $=$ recursive partitioning analysis; $\mathrm{RTOG}=$ Radiation Therapy Oncology Group; SRS = stereotactic radiosurgery; WBRT = wholebrain radiotherapy.
}

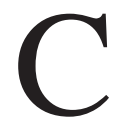
URRENT evidence-based guidelines have supported the use of SRS for patients with $1-4$ brain metastases (METs). ${ }^{21}$ However, as Sheehan and Schlesinger ${ }^{29}$ very recently stated, such guidelines frequently lag behind contemporary clinical practice because 
several years, at least, are required for conducting rigorous prospective clinical trials. In fact, as we reported previously ${ }^{36,37}$ and as described by Knisely et al., ${ }^{20}$ a trend for patients with $\geq 5$, or even $\geq 10$, tumors to be potential candidates for SRS alone had already become apparent in the early 21 st century. Tsao et al. ${ }^{33}$ recently stated, in the American Society for Radiation Oncology evidencebased guideline, that "when new brain METs are seen on the planning scan the day of SRS, it may be reasonable to proceed and complete the SRS procedure for all of the lesions visualized even if they exceed a total of 4 brain METs." Also, Grandhi et al..$^{10}$ very recently reported that SRS can be used to safely and effectively treat intracranial disease with a high rate of local control in patients with $\geq$ 10 brain METs.

In 1997, the first author (M.Y.) reported 2 lung cancer patients in whom more than 30 brain METs were successfully controlled for 4.5 and 5.5 months (the respective remaining survival periods after SRS alone). ${ }^{35}$ Although retrospective studies of SRS-treated patients with many brain METs have since been reported, these studies were based on small patient numbers. 3,6,14,19,30,32 Therefore, the role of SRS for patients with $\geq 5$ brain METs has not yet been sufficiently analyzed based on databases with a large sample size. The goals of this retrospective cohort study, based on our patients with SRS-treated brain METs, were to reappraise whether treatment results were truly inferior for tumor numbers of $\geq 5$ versus $1-4$ and to identify factors determining inferiority and/or noninferiority.

\section{Methods}

\section{Patient Population}

This institutional review board-approved, retrospective cohort study used our prospectively accumulated database at Tokyo Women's Medical University, including 2553 consecutive patients. Patients in our series underwent SRS alone, not in combination with concurrent WBRT, for brain METs during the 13-year period between July 1998 and June 2011. As all patients had been referred to us for SRS, their primary physicians had mostly made the patient selections. Patient selection criteria may thus have differed among referring physicians. Therefore, one author (M.Y.) decided whether to accept a patient. We did not perform SRS on patients with low KPS ${ }^{18}$ scores due to systemic diseases $(<70 \%)$, a uncooperative state due to poor neurocognitive function, meningeal dissemination, or an anticipated survival period of 3 months or less. Therefore, only 173 patients $(6.8 \%)$ were categorized into RPA Class $3{ }^{8}$ Table 1 summarizes clinical characteristics of the entire cohort and also for Group A (1-4 tumors, 1553 patients) and Group B ( $\geq 5$ tumors, 1000 patients). Because all patients had been referred to us for SRS by other facilities, the primary physicians responsible for each patient decided the indications for both surgery and radiotherapy. Therefore, prior to SRS, $18.3 \%$ of the 2553 patients had undergone surgical removal of brain METs and $4.8 \%$ had undergone WBRT (Table 1).

The treatment strategy was explained in detail to each patient, and at least one adult relative, by the first author (M.Y.) before SRS. Written informed consent was obtained from all patients. Our previous report described our radiosurgical techniques in detail. ${ }^{37}$ Briefly, standard SRS procedures were performed using a Leksell Gamma Unit model B before June 2003 and thereafter a Leksell Gamma Unit model C (Elekta AB). Regarding dose selection in cases with multiple METs, total absorbed energy to the whole skull $<15$ Joules was considered to be safe, as we have reported elsewhere..$^{36,37}$ According to this upper limit criterion, a peripheral dose of $22 \pm 3$ Gy was applied to cases in which cumulative tumor volumes did not exceed $10.0 \mathrm{~cm}^{3}$, while those $>10.0 \mathrm{~cm}^{3}$ received $18 \pm 3$ Gy. Furthermore, a tumor with a maximum lesion volume $>10.0 \mathrm{~cm}^{3}$ was irradiated with $18 \mathrm{~Gy}$ or less. Irradiation doses to the optic apparatus should not exceed 10-12 Gy. In cases with brainstem lesions, a peripheral dose of 18-20 Gy can be used for tumor volumes < 1 $\mathrm{cm}^{3}, 16-18$ Gy for $1-4 \mathrm{~cm}^{3}$, and no more than 15 Gy for $>4 \mathrm{~cm}^{3}$. In the few patients (4.8\%) who had undergone WBRT, peripheral doses were decreased by $10 \%-15 \%$. After SRS, all cases were routinely managed by referring physicians, and patients were recommended to have clinical and neuroimaging examinations at an approximately 2- to 3-month interval. However, in 760 (29.8\%) of the 2553 patients, neuroimaging follow-up could not be performed due to early post-SRS death or remarkable deterioration of general condition. Approximately $50 \%$ of patients came to our outpatient clinic periodically, while clinical and/or neuroimaging data were sent to us by mail in about $25 \%$. The first author (M.Y.) called the remaining $25 \%$ of patients or their relatives to confirm patients' conditions. For cases in which patients had died, the day of death, cause of death, and detailed information on condition changes were surveyed by telephone.

\section{Case Matching}

As shown in Table 1, there was considerable bias between Groups A and B. Therefore, a case-matched study was conducted by one of the authors (Y.S.), who did not participate in other aspects of this study and was blinded to final outcomes. Patient selection was performed by employing the propensity score matching method with a Greedy 5-To-1 Digit-Matching algorithm for clinical factors, (that is, age, sex, primary tumor state, extracerebral METs, KPS score, neurological symptoms, prior procedures [surgery and WBRT], volume of the largest tumor, and peripheral doses). ${ }^{5,25}$

\section{Clinical Outcomes}

The primary end point was overall survival, and the secondary end points were neurological death, neurological deterioration, local recurrence of the treated tumor, repeat SRS for new lesions, and SRS-induced major complications. For each end point, failures were regarded as events and any others as censored. Overall survival time was defined as the interval between the first SRS and death due to any cause (that is, progression of systemic and/or brain METs, other cancer-unrelated diseases, accident, suicide, and so on, or the day of the last follow-up). Neurological death was defined as death caused by any 
M. Yamamoto et al.

TABLE 1: Summary of clinical characteristics of 2553 patients with brain METs

\begin{tabular}{|c|c|c|c|c|}
\hline \multirow[b]{2}{*}{ Characteristic } & \multirow[b]{2}{*}{ Total } & \multicolumn{2}{|c|}{ Tumor Nos. } & \multirow[b]{2}{*}{$p$ Value* } \\
\hline & & 1-4 (Group A) & $\geq 5$ (Group B) & \\
\hline no. of patients & 2553 & 1553 & 1000 & \\
\hline \multicolumn{5}{|l|}{ tumor no. } \\
\hline median & 3 & 2 & 10 & \\
\hline range & $1-89$ & $1-4$ & $5-89$ & \\
\hline \multicolumn{5}{|l|}{ age (yrs) } \\
\hline median & 64.0 & 64.7 & 63.0 & 0.001 \\
\hline range & $19-96$ & $19-96$ & $19-91$ & \\
\hline \multicolumn{5}{|l|}{ sex } \\
\hline female & 1004 & 557 & 447 & \\
\hline male & 1549 & $996(64.1 \%)$ & $553(55.3 \%)$ & $<0.001$ \\
\hline \multicolumn{5}{|l|}{ primary cancer sites } \\
\hline lung & 1658 & $974(62.7 \%)$ & $684(68.4 \%)$ & $0.011 \dagger$ \\
\hline alimentary tract & 302 & $225(14.5 \%)$ & $76(7.6 \%)$ & \\
\hline breast & 279 & $131(8.4 \%)$ & $148(14.8 \%)$ & \\
\hline kidney & 103 & $79(5.1 \%)$ & $24(2.4 \%)$ & \\
\hline melanoma & 15 & $10(0.6 \%)$ & $5(0.5 \%)$ & \\
\hline others & 169 & $134(8.6 \%)$ & $63(6.3 \%)$ & \\
\hline \multicolumn{5}{|c|}{ primary cancer status } \\
\hline controlled & 738 & 493 & 245 & \\
\hline not controlled & 1815 & $1060(68.3 \%)$ & $755(75.5 \%)$ & 0.001 \\
\hline \multicolumn{5}{|l|}{ extracerebral METs } \\
\hline no & 1332 & 848 & 484 & \\
\hline yes & 1221 & 705 (45.4\%) & $516(51.6 \%)$ & 0.002 \\
\hline \multicolumn{5}{|l|}{ KPS score } \\
\hline$\geq 80 \%$ & 1945 & 1209 & 736 & \\
\hline$\leq 70 \%$ & 608 & $344(22.2 \%)$ & $264(26.4 \%)$ & 0.015 \\
\hline \multicolumn{5}{|l|}{ RPA class } \\
\hline 1 & 195 & 145 & 50 & \\
\hline 2 & 2185 & $1324(85.3 \%)$ & $861(86.1 \%)$ & $0.42 \ddagger$ \\
\hline 3 & 173 & 84 & 89 & \\
\hline \multicolumn{5}{|c|}{ neurological symptoms } \\
\hline no & 1234 & 742 & 510 & \\
\hline yes & 1319 & $829(53.4 \%)$ & $490(49.0 \%)$ & 0.031 \\
\hline \multicolumn{5}{|l|}{ prior surgery } \\
\hline no & 2085 & 1226 & 859 & \\
\hline yes & 468 & 327 (21.1\%) & $141(14.0 \%)$ & $<0.001$ \\
\hline \multicolumn{5}{|l|}{ prior WBRT } \\
\hline no & 2429 & 1496 & 933 & \\
\hline yes & 122 & 57 (3.7\%) & $65(6.5 \%)$ & 0.001 \\
\hline \multicolumn{5}{|l|}{ tumor vol $\left(\mathrm{cm}^{3}\right)$} \\
\hline \multicolumn{5}{|l|}{ cumulative } \\
\hline mean & 9.92 & 8.71 & 11.80 & $<0.001$ \\
\hline range & $0.01-126.2$ & $0.01-126.2$ & $0.10-115.3$ & \\
\hline \multicolumn{5}{|l|}{ largest tumor } \\
\hline mean & 6.92 & 7.55 & 5.93 & $<0.001$ \\
\hline range & $0.01-94.2$ & $0.01-89.3$ & $0.03-94.2$ & \\
\hline
\end{tabular}

(continued) 
TABLE 1: Summary of clinical characteristics of 2553 patients with brain METs (continued)

\begin{tabular}{|c|c|c|c|c|}
\hline \multirow[b]{2}{*}{ Characteristic } & \multirow[b]{2}{*}{ Total } & \multicolumn{2}{|c|}{ Tumor Nos. } & \multirow[b]{2}{*}{ p Value* } \\
\hline & & 1-4 (Group A) & $\geq 5$ (Group B) & \\
\hline \multicolumn{5}{|l|}{ peripheral dose (Gy) } \\
\hline mean & 21.14 & 21.67 & 20.33 & $<0.001$ \\
\hline range & $10.00-32.00$ & $10.00-32.00$ & $10.00-27.00$ & \\
\hline
\end{tabular}

intracranial disease (that is, tumor recurrence, carcinomatous meningitis, cerebral dissemination, and progression of other untreated intracranial tumors).

Local recurrence-free survival time was defined as the interval between the first SRS and the day when follow-up MR imaging demonstrated local recurrence (at the irradiated lesion). Generally, local recurrence criteria were increased size of an enhanced area on post-Gd T1-weighted MR images and enlarged tumor core on T2weighted MR images. ${ }^{15}$ However, in 115 cases in which MRI alone was not sufficient to confirm recurrence, ${ }^{11} \mathrm{C}$ methionine PET was used to distinguish tumor recurrence from necrotic lesions. ${ }^{22,23,34,38}$ Positron emission tomography was performed, and the results were evaluated by one author (T.N.) not involved in either SRS treatment or patient follow-up. Thus, all findings of recurrence on MRI and/or PET were regarded as events and any others as censored. Also, repeat SRS-free survival time was defined as the interval between the first SRS and the day the second SRS was performed for new METs; all repeat SRS procedures for newly developed lesions were regarded as events and any others as censored. For patients developing new brain METs after the first SRS, our approach is similar to that in patients with initially diagnosed brain METs. As to tumor size, if follow-up MRI demonstrates tumors with diameters of $2-3 \mathrm{~mm}$ in the brainstem or optic apparatus, we perform repeat SRS without further observation. Otherwise, repeat SRS is usually postponed with close MRI follow-up until the tumor diameter exceeds approximately $1 \mathrm{~cm}$.

Neurological deterioration-free survival time was defined as the interval between the first SRS and the day that any brain disease-caused neurological worsening manifested (that is, local recurrence, progression of new lesions, and SRS-induced complications). Decreases in KPS scores, in patients with scores $\geq 20 \%$, due to neurological worsening were regarded as events and any others as censored. Major complication-free survival time was taken as the interval between the first SRS and the day major SRS-induced complications occurred. Patients with major complications included those with RTOG neurotoxicity grades of 2 or worse and, even if the grade was either 0 or 1 , those in whom surgical intervention was required based on sequential MRI follow-up demonstrating progressive enlargement of a cyst and/or a mass lesion with further observation thus being regarded as excessively high risk; all of these conditions were regarded as events and any others as censored. ${ }^{24}$

\section{Statistical Analysis}

All data were analyzed according to the intentionto-treat principle. For the baseline variables, summary statistics were constructed by using frequencies and proportions for categorical data and means \pm SD for continuous variables. We compared patient characteristics using the Fisher exact test for categorical outcomes and t-tests for continuous variables, as appropriate. The standard Kaplan-Meier method was used for overall and neurological death-free survivals. ${ }^{16}$ Also, univariate analysis using the Cox proportional hazard model was performed to determine pre-SRS clinical factors favoring longer survival. ${ }^{4}$

For time-to-event outcomes, the cumulative incidences of local recurrence, repeat SRS, neurological deterioration, and major complications were estimated by a competing risk analysis, because death is a competing risk for loss to follow-up (that is, patients who die can no longer become lost to follow-up)..$^{9,11,26}$ Also, to identify baseline and clinical variables associated with the 4 aforementioned outcomes, competing risk analyses were performed with the Fine-Gray generalization of the proportional hazards model accounting for death as a competing risk. ${ }^{7}$ Fine-Gray generalization makes use of the subdistribution hazard to model cumulative incidence, thereby quantifying the overall benefit or harm of an exposure. $^{2}$

All comparisons were planned, and the tests were 2 -sided. A p value $<0.05$ was considered to be statistically significant. All statistical analyses were performed by one of the authors (Y.S.) using SAS software version 9.2 (SAS Institute) and the R statistical program, version 2.13. Before statistical analyses, the database was cleaned (by Y.H.). These two authors were not involved in either SRS treatment or patient follow-up.

\section{Results}

\section{Cohort Study}

Four patients ( $0.15 \%$; 1 in Group A and 3 in Group B) were lost to follow-up. As of the end of December 2011, 201 patients $(7.9 \%)$ were confirmed to be alive (censored observation) and the remaining 2348 (92.0\%) had died (event). The mean post-SRS follow-up periods were 36.1 months (95\% CI 31.6-40.5 months) in the censored subgroup and 10.4 months (95\% CI 10.0-10.9 months) in the event subgroup; the overall mean post-SRS follow-up du- 
ration was 12.5 months (95\% CI 11.8-13.1 months). The MST after SRS was 7.4 months (95\% CI 7.1-7.9 months). Cumulative post-SRS survival rates were $57.6 \%, 33.7 \%$, $15.0 \%, 8.3 \%$, and $4.5 \%$ at the 6th, 12th, 24th, 36th, and 60 th post-SRS month, respectively. Causes of death could not be determined in 106 patients but were confirmed in the remaining 2242 to be nonbrain diseases in 1983 $(88.4 \%)$ and brain diseases in $259(11.6 \%)$.

Among various pre-SRS clinical factors, univariate analysis demonstrated age $\leq 65$ years, female sex, KPS score $\geq 80 \%$, cumulative tumor volume $\leq 10 \mathrm{~cm}^{3}$, controlled primary cancer, no extracerebral METs, and neurologically asymptomatic status to be significant factors favoring longer survival, as shown in Table 2. For all clinical factors significantly impacting survival, both hazard ratios and probability values were very similar in the 2 groups.

\section{Case-Matched Study}

As described above, after all the propensity-score matches had been performed, we compared baseline covariates between the 2 groups. Ultimately, 1096 patients (548 with 1-4 tumors [Group A] and 548 with $\geq 5$ tumors [Group B]) were selected. The $\mathrm{p}$ values after matching were $>0.05$ for all clinical factors (Table 3 ).

As shown in Fig. 1 left, MST after SRS was significantly longer in the 548 patients in Group A than in the 548 in Group B (7.9 vs 7.0 months, HR 1.176 , 95\% CI $1.039-1.331, \mathrm{p}=0.01)$. However, incidences of death caused by progression of brain disease were very similar: $10.6 \%$ in Group A and $8.2 \%$ in Group B $(p=0.21)$ (Table 4). Furthermore, there was no significant difference between the 2 groups in neurological death-free survival intervals (HR 0.945, 95\% CI 0.636-1.394, p = 0.77) (Fig. 1 right).
Post-stereotactic radiosurgery follow-up MRI examinations were available in 763 patients (69.6\%): 378 in Group A and 385 in Group B. Among these 763 patients, the incidence of local recurrence was significantly higher in the Group A than in the Group B patients $(8.5 \%$ vs $3.9 \%, p=0.01)($ Table 4$)$. Nevertheless, there was no significant difference between the 2 groups in local recurrence-free survival intervals (HR $0.577,95 \%$ CI $0.312-$ $1.069, \mathrm{p}=0.08)$ (Fig. 2A).

As shown in Table 4, there were no significant differences between Groups A and B in the incidences of salvage WBRT (3.7\% vs 5.8\%, $\mathrm{p}=0.11)$, salvage surgery (2.2\% vs $1.1 \%, p=0.23)$, repeat SRS for new lesions (30.3\% vs $29.0 \%, \mathrm{p}=0.69)$, neurological deterioration (13.1\% vs $9.7 \%, \mathrm{p}=0.09$ ), or SRS-related complications ( $2.7 \%$ vs $2.0 \%, \mathrm{p}=0.55)$. Also, there were no significant differences between the 2 groups in the repeat SRS-free survival intervals (HR 1.133, 95\% CI 0.910-1.409, $\mathrm{p}=$ 0.26) (Fig. 2B), neurological deterioration-free survivals (HR 1.868, 95\% CI 0.608-1.240, p = 0.44) (Fig. 2C), or SRS-related complication-free survival intervals (HR $1.105,95 \%$ CI $0.490-2.496, p=0.81$ ) (Fig. 2D), all of which were estimated using competing risk analysis.

\section{Discussion}

\section{Do Tumor Numbers Impact Post-SRS Treatment Results?}

At present, the majority of physicians consider patients with 1-4 METs to be good candidates for SRS with or without WBRT. Debate continues as to how many brain METs make a patient ineligible for SRS alone. Karlsson et al. ${ }^{17}$ reported, based on 1921 MET patients who underwent SRS, that despite patients with a single MET sur-

TABLE 2: Clinical factors before SRS impacting post-SRS survival period in 2549 patients*

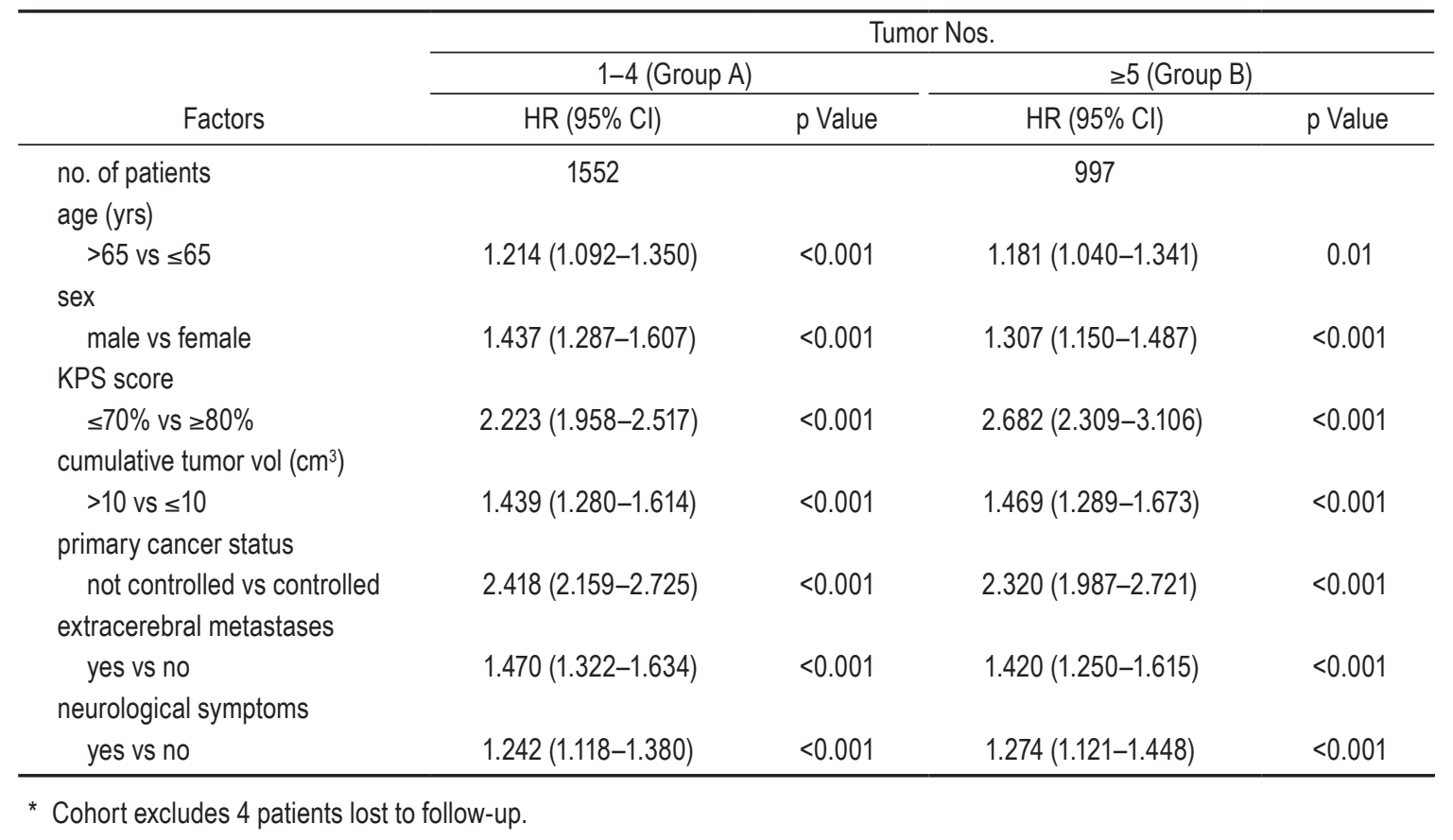


Radiosurgery for $1-4$ vs $\geq 5$ brain metastases

TABLE 3: Summary of clinical characteristics of 1096 case-matched patients with brain METs

\begin{tabular}{|c|c|c|c|c|}
\hline \multirow[b]{2}{*}{ Characteristic } & \multirow[b]{2}{*}{ Total } & \multicolumn{2}{|c|}{ Tumor Nos. } & \multirow[b]{2}{*}{ p Value* } \\
\hline & & 1-4 (Group A) & $\geq 5$ (Group B) & \\
\hline no. of patients & 1096 & 548 & 548 & \\
\hline \multicolumn{5}{|l|}{ tumor no. } \\
\hline median & 4 & 2 & 8 & \\
\hline range & $1-51$ & $1-4$ & $5-51$ & \\
\hline \multicolumn{5}{|l|}{ age (yrs) } \\
\hline median & 63.8 & 63.7 & 63.9 & 0.7 \\
\hline range & $19-91$ & $19-88$ & $19-91$ & \\
\hline \multicolumn{5}{|l|}{ sex } \\
\hline female & 465 & 240 & 225 & \\
\hline male & 631 & $308(56.2 \%)$ & $323(58.9 \%)$ & 0.36 \\
\hline \multicolumn{5}{|l|}{ primary cancer sites } \\
\hline lung & 737 & $366(66.8 \%)$ & $371(67.7 \%)$ & $0.75 \dagger$ \\
\hline alimentary tract & 108 & $55(10.0 \%)$ & $53(9.7 \%)$ & \\
\hline breast & 135 & $68(12.4 \%)$ & $67(12.2 \%)$ & \\
\hline kidney & 34 & $15(2.7 \%)$ & $19(3.5 \%)$ & \\
\hline melanoma & 6 & $2(0.4 \%)$ & $4(0.7 \%)$ & \\
\hline others & 76 & $42(7.7 \%)$ & $34(6.2 \%)$ & \\
\hline \multicolumn{5}{|l|}{ primary cancer status } \\
\hline controlled & 299 & 145 & 154 & \\
\hline not controlled & 797 & $403(73.5 \%)$ & $394(71.9 \%)$ & 0.54 \\
\hline \multicolumn{5}{|l|}{ extracerebral METs } \\
\hline no & 575 & 283 & 292 & \\
\hline yes & 521 & $265(48.4 \%)$ & $256(46.7 \%)$ & 0.59 \\
\hline \multicolumn{5}{|l|}{ KPS score } \\
\hline$\geq 80 \%$ & 848 & 412 & 436 & \\
\hline$\leq 70 \%$ & 248 & $136(24.8 \%)$ & $112(20.4 \%)$ & 0.08 \\
\hline \multicolumn{5}{|l|}{ RPA class } \\
\hline 1 & 75 & 43 & 32 & \\
\hline 2 & 949 & $468(85.4 \%)$ & $481(87.8 \%)$ & $0.19 \ddagger$ \\
\hline 3 & 72 & 37 & 35 & \\
\hline \multicolumn{5}{|c|}{ neurological symptoms } \\
\hline no & 561 & 286 & 275 & \\
\hline yes & 535 & $262(47.8 \%)$ & $273(49.8 \%)$ & 0.51 \\
\hline \multicolumn{5}{|l|}{ prior surgery } \\
\hline no & 914 & 461 & 453 & \\
\hline yes & 182 & 87 (15.9\%) & $95(17.3 \%)$ & 0.52 \\
\hline \multicolumn{5}{|l|}{ prior WBRT } \\
\hline no & 1048 & 524 & 524 & \\
\hline yes & 46 & $24(4.4 \%)$ & $22(4.0 \%)$ & 0.24 \\
\hline \multicolumn{5}{|l|}{ tumor vol $\left(\mathrm{cm}^{3}\right)$} \\
\hline \multicolumn{5}{|l|}{ cumulative } \\
\hline mean & 9.02 & 9.12 & 8.92 & 0.79 \\
\hline range & $0.01-122.2$ & $0.01-122.0$ & $0.10-115.3$ & \\
\hline \multicolumn{5}{|l|}{ largest tumor } \\
\hline mean & 6.39 & 6.84 & 5.95 & 0.09 \\
\hline range & $0.01-94.2$ & $0.01-89.3$ & $0.03-94.2$ & \\
\hline
\end{tabular}

(continued) 
M. Yamamoto et al.

TABLE 3: Summary of clinical characteristics of 1096 case-matched patients with brain METs (continued)

\begin{tabular}{|c|c|c|c|c|}
\hline \multirow[b]{2}{*}{ Characteristic } & \multirow[b]{2}{*}{ Total } & \multicolumn{2}{|c|}{ Tumor Nos. } & \multirow[b]{2}{*}{ p Value* } \\
\hline & & 1-4 (Group A) & $\geq 5$ (Group B) & \\
\hline \multicolumn{5}{|l|}{ peripheral dose (Gy) } \\
\hline mean & 21.51 & 21.44 & 21.58 & 0.42 \\
\hline range & $10.00-27.00$ & $12.00-25.00$ & $10.00-27.00$ & \\
\hline
\end{tabular}

viving longer than those with multiple METs, there were no significant MST differences among individuals with 2, $3-4,5-8$, or $>8$ metastases. Chang et al. ${ }^{3}$ recently reported, based on 323 SRS-treated patients with brain METs, that there were no significant MST differences among 4 tumor number groups (that is, 1-5, 6-10, 11-15, and > 15). The first author (M.Y.) has described elsewhere that the Kaplan-Meier method was used to compare 15 pairs of groups based on tumor numbers: 1 vs $\geq 2, \leq 2$ vs $\geq 3$, and so on through $\leq 15$ vs $\geq 16$. In each of the 15 pairs, the MSTs in patients with lower tumor numbers were significantly longer than those in patients with higher tumor numbers $(p<0.0001) .{ }^{37}$ Furthermore, 14 other pairs of groups, based on tumor numbers, were also assessed by this method ( 1 vs 2, 2 vs 3, 3 vs 4, and so on through 14 vs 15). Among the 14 pairs, only 1 vs 2 showed a significant MST difference $(p=0.0002)$; no significant differences were detected for the other 13 pairs. ${ }^{37}$

In our present study, although the post-SRS MST difference, 0.9 months, between the 2 groups was statistically significant, this difference was not clinically meaningful. Furthermore, approximately $90 \%$ of patients with brain METs died of causes other than brain disease progression, regardless of tumor number, when only carefully selected patients were treated. Also, as mentioned, the Group B patients were demonstrated to have noninferior results compared with the Group A patients, in terms of neurological death, local recurrence, repeat SRS required for new tumors, maintenance of good neurological state, and SRS-related complications. Particularly, as reported previously, ${ }^{36}$ SRS for patients with multiple METs was not found to be excessively high risk in carefully selected patients. Furthermore, we also reported very recently that, based on 167 patients surviving more than 3 years after SRS, tumor numbers did not impact the incidence of SRS-induced complications (HR 1.066, 95\% CI 0.968$1.131, \mathrm{p}=0.1567) .{ }^{38}$ Our current results showed no apparent increase in the risk of complications with SRS for $\geq 5$ METs compared with 1-4 METs. Furthermore, post-SRS MRI confirmed the absence of leukoencephalopathy in patients receiving SRS alone.

Because approximately $90 \%$ of patients died due to extracerebral diseases, it is clearly crucial for brain MET treatments to maintain a good neurological state in those patients treated. We thus consider it to be very important that the currently reported SRS results of good neurological status maintenance in patients with $\geq 5$ tumors were clearly noninferior to those in patients with 1-4 tumors.

\section{Is WBRT Necessary for All Patients With Multiple METs?}

The central criticism of using SRS alone for multiple METs is the assumption that frequent microscopic tumors will soon require salvage SRS or other treatment. Thus, WBRT has generally been advocated. However, WBRT can be expected to prevent new tumors arising within 6-8 post-WBRT months at the longest, as shown in Fig. 2 of the article written by Aoyama et al. ${ }^{1}$ We should remember that considerable numbers of patients with brain METs can survive more than 1 year, outliving the effects of
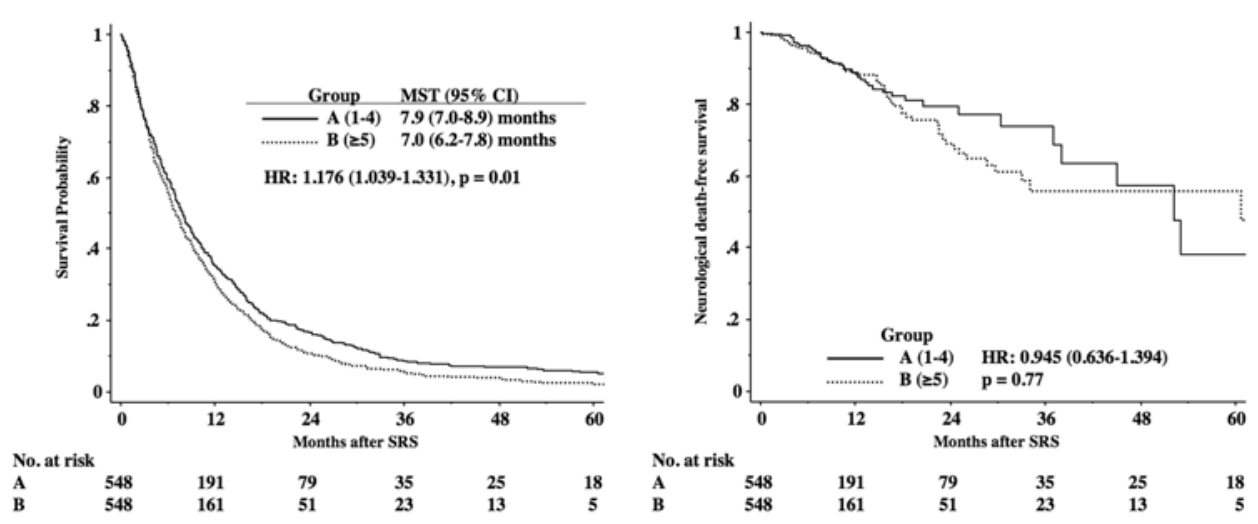

FIG. 1. Overall survival (left) and neurological death-free survival (right), based on a subset of 1096 case-matched patients according to tumor number (1-4 [Group A] and $\geq 5$ [Group B]), estimated using the standard Kaplan-Meier method. 
TABLE 4: Summary of treatment results after SRS

\begin{tabular}{lrccr}
\hline \multirow{2}{*}{ Incidences } & Total & $1-4($ Group A) & $\geq 5$ (Group B) & p Value \\
\cline { 3 - 4 } \cline { 3 - 4 } no. of patients & 1096 & 548 & 548 & \\
neurological death & 103 & $58(10.6 \%)$ & $45(8.2 \%)$ & 0.21 \\
salvage WBRT & 52 & $20(3.7 \%)$ & $32(5.8 \%)$ & 0.11 \\
salvage surgery & 18 & $12(2.2 \%)$ & $6(1.1 \%)$ & 0.23 \\
local recurrence* & 47 & $32(8.5 \%)$ & $15(3.9 \%)$ & 0.01 \\
repeat SRS & 325 & $166(30.3 \%)$ & $159(29.0 \%)$ & 0.69 \\
neurological deterio- & 125 & $72(13.1 \%)$ & $53(9.7 \%)$ & 0.09 \\
$\quad$ ration & & & & \\
$\quad$ SRS-related compli- & 26 & $15(2.7 \%)$ & $11(2.0 \%)$ & 0.55 \\
$\quad$ cations & & & & \\
\hline
\end{tabular}

* Based on 763 patients (378 in Group A and 385 in Group B; 333 were excluded because neuroimaging results were not available).

WBRT. Most fortunately, we already live in an era when an MET with a diameter of $2 \mathrm{~mm}$ or even slightly smaller can be detected using thin-slice, postenhanced MR images. ${ }^{13}$ Hanssens et al. ${ }^{12}$ recently reported that SRS alone based on high-resolution MRI decreased the incidence of and lengthened the time to distant recurrences. In fact, although data on periods between SRS and the appearance of new lesions were not available, the present study showed that the repeat-SRS rate in our Group B patients $(29.0 \%)$ was very similar to that in our Group A patients $(30.3 \%, p=0.69)$, as shown in Table 4. Furthermore, we found that the repeat-SRS-free intervals (when SRS was performed for new lesions) were almost the same for our Group A and Group B patients (Fig. 2B). Therefore, the availability of an alternative treatment for multiple brain METs allows WBRT to be reserved for subsequent treatment attempts (that is, for meningeal dissemination or miliary METs treatable only with WBRT).

Most physicians consider current evidence to clearly support WBRT use over SRS for patients with poor performance status and progressive/uncontrolled systemic disease and, ultimately, a relatively short survival expectancy. ${ }^{1}$ Therefore, as described in Patient Population, we usually do not perform SRS in patients with low KPS ${ }^{18}$ scores due to systemic diseases $(<70 \%)$ and an anticipated survival period of 3 months or less. However, in other patients, the availability of an alternative treatment for brain METs allows WBRT to be postponed relative to the course of another management strategy, such as very extensive chemotherapy and/or radiation therapy for spinal
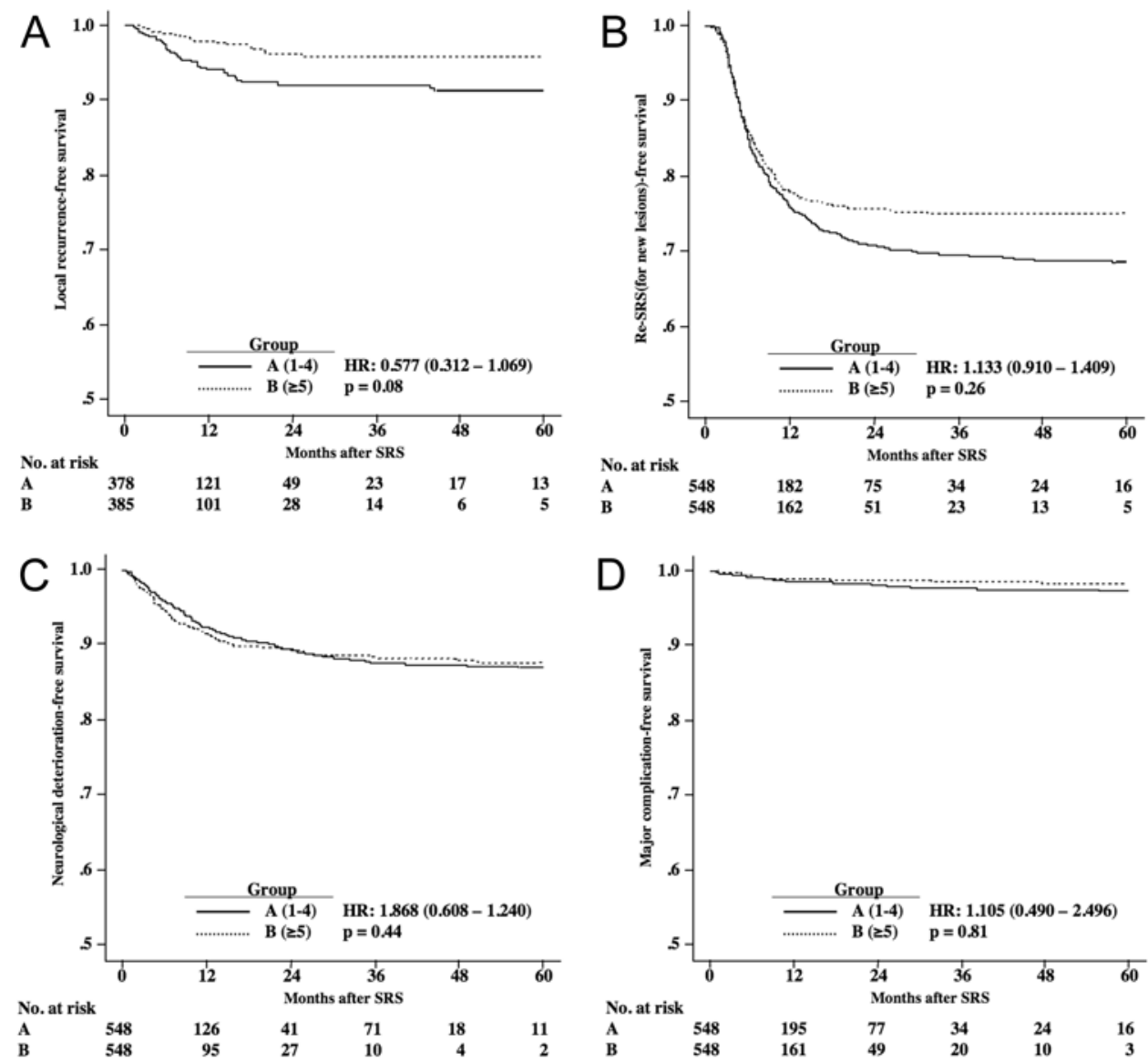

FIG. 2. Local recurrence-free survival (A), repeat SRS (for new lesions)-free survival (B), neurological deterioration-free survival (C), and major complication-free survival (D) according to tumor number (1-4 [Group A] and $\geq 5$ [Group B]) estimated using competing risk analysis (see text). 
lesions or other organ involvement, which are also urgent. Furthermore, SRS takes only 1 day, whereas $2-3$ weeks are necessary for completing WBRT. Thus, SRS allows patients, and this is especially important for those with a short survival expectancy, to maximize any remaining time with their families.

The North American Gamma Knife Consortium is currently conducting a prospective randomized study entitled "Neurocognitive outcomes in patients treated with radiotherapy for five or more brain metastases (NAGKCRand)" (Identifier NCI01731704; http://www.clinicaltrials. gov/). The primary aim of this study is to compare the change in neurocognitive function outcomes between baseline and 6 months in WBRT versus SRS treatment groups. Patients with $\geq 5$ METs are selected for this study. The results of this study are expected to clarify the role of SRS alone versus WBRT.

\section{Weaknesses of the Present Study}

As mentioned in our previous article, ${ }^{39}$ in general the major weakness of a retrospective study might be that clinical factors are obviously heterogeneous. In fact, there was considerable bias between Groups A and B in our cohort (Table 1). Greater patient group homogeneity makes a study more scientific. However, heterogeneity actually reflects clinical settings rather closely, as we physicians often deal with inhomogeneous clinical factors. In particular, our database included some patients whose brain METs were not newly diagnosed tumors. However, proportions of such patients in the 2 groups were very small and did not differ significantly (Table 3). Thus, this heterogeneity had only a minimal impact on our results, as we reported very recently. ${ }^{31}$ Nevertheless, treatment selection is considered to be largely influenced by the characteristics of patients receiving a particular treatment regimen. This is an important issue when estimating the effect of treatments or exposures on outcomes using observational data. One approach to reducing or eliminating the effect of treatment selection bias and confounding effects is to use propensity score matching, which allows one to design and analyze an observational (nonrandomized) study that mimics some of the characteristics of a randomized controlled trial. Therefore, in the present investigation, a case-matched study was also conducted by one of the authors (Y.S.), who did not participate in other aspects of this study and was blinded to final outcomes.

Only patients with RTOG neurotoxicity Grade 2 or worse were counted in this study because, if severe problems, not only those that were symptomatic but also those shown only on MRI, occurred in SRS-treated patients, every physician, without exception, consulted the first author. In fact, some busy physicians actually forgot to report minor problems like RTOG neurotoxicity Grade 0 or 1 to us. Therefore, a weakness of this study is that all patients with minor complications were not surveyed.

\section{Ongoing Prospective Cohort Study}

The Japanese Leksell Gamma Knife Society is currently conducting a prospective observational study en- titled "Gamma knife treatment results for patients with multiple brain metastases: A multi-institutional prospective study" (abbreviation JLGK0901; trial no. 1812; http:// www.umin.ac.jp/)." This investigation was designed to examine whether SRS alone for patients with 5-10 brain METs is not inferior to SRS alone for patients with 2-4 METs in terms of overall survival and other clinical results. Although the final result of this ongoing study is due in early 2013, based on our previous retrospective investigations, the JLGK0901 study is anticipated to show noninferiority of SRS as the sole treatment for patients with 5-10 brain METs compared with 2-4 METs in terms of overall survival. ${ }^{27,28}$ However, a randomized controlled trial, in the near future, is necessary to clarify the most appropriate role for SRS alone in patients with $\geq 5$ METs.

\section{How Should Good Candidates for SRS Alone Be Selected From Among Patients With $\geq 10$ METs?}

The selection of good candidates for SRS alone, even from among patients with $\geq 5$ METs, is a very important issue. As shown in Table 2, we identified pre-SRS clinical factors that significantly favored longer survival after SRS. Among these factors, KPS score $\geq 80 \%$ and controlled primary cancer were regarded as the 2 major prognostic factors for selecting good candidates. It must be noted that both hazard ratios and probability values for the 7 clinical factors were very similar in the 2 groups. This means that it is not necessary for physicians to use different patient selection criteria when they manage patients with $\geq 5$ METs.

\section{Conclusions}

Although our retrospective study suggests increased tumor number to be an unfavorable factor for longer survival, the post-SRS MST difference, 0.9 months, between the 2 groups is not clinically meaningful. Furthermore, patients with $\geq 5$ METs have noninferior results compared with patients with 1-4 METs, in terms of neurological death, local recurrence, repeat SRS, maintenance of a good neurological state, and SRS-related complications. A randomized controlled trial should be conducted to test this hypothesis.

\section{Disclosure}

The authors report no conflict of interest concerning the materials or methods used in this study or the findings specified in this paper.

Author contributions to the study and manuscript preparation include the following. Conception and design: Yamamoto. Acquisition of data: Yamamoto, Kawabe. Analysis and interpretation of data: Yamamoto. Drafting the article: Yamamoto, Barfod. Critically revising the article: all authors. Reviewed submitted version of manuscript: all authors. Approved the final version of the manuscript on behalf of all authors: Yamamoto. Statistical analysis: Sato, Higuchi. Study supervision: Yamamoto, Kasuya. Evaluation of PET findings: Nariai. Checked English in manuscript: Barfod. General patient care: Urakawa.

\section{Acknowledgment}

The authors are very grateful to L. Dade Lunsford, M.D., 
Department of Neurosurgery, University of Pittsburgh Medical Center, for his outstanding help in preparing this manuscript.

\section{References}

1. Aoyama H, Shirato H, Tago M, Nakagawa K, Toyoda T, Hatano K, et al: Stereotactic radiosurgery plus whole-brain radiation therapy vs stereotactic radiosurgery alone for treatment of brain metastases: a randomized controlled trial. JAMA 295:2483-2491, 2006

2. Bakoyannis G, Touloumi G: Practical methods for competing risks data: a review. Stat Methods Med Res 21:257-272, 2012

3. Chang WS, Kim HY, Chang JW, Park YG, Chang JH: Analysis of radiosurgical results in patients with brain metastases according to the number of brain lesions: is stereotactic radiosurgery effective for multiple brain metastases? Clinical article. J Neurosurg 113 Suppl:73-78, 2010

4. Cox DR: Regression models and life tables. J R Stat Soc B 34:187-220, 1972

5. D'Agostino RB Jr: Propensity score methods for bias reduction in the comparison of a treatment to a non-randomized control group. Stat Med 17:2265-2281, 1998

6. DiLuna ML, King JT Jr, Knisely JPS, Chiang VL: Prognostic factors for survival after stereotactic radiosurgery vary with the number of cerebral metastases. Cancer 109:135-145, 2007

7. Fine JP, Gray RJ: A: proportional hazards model for the subdistribution of a competing risk. J Am Stat Assoc 94:496509, 1999

8. Gaspar L, Scott C, Rotman M, Asbell S, Phillips T, Wasserman $\mathrm{T}$, et al: Recursive partitioning analysis (RPA) of prognostic factors in three Radiation Therapy Oncology Group (RTOG) brain metastases trials. Int J Radiat Oncol Biol Phys 37:745751,1997

9. Gooley TA, Leisenring W, Crowley J, Storer BE: Estimation of failure probabilities in the presence of competing risks: new representations of old estimators. Stat Med 18:695-706, 1999

10. Grandhi R, Kondziolka D, Panczykowski, D, Monaco EA, Kano H, Niranjan A, et al: Stereotactic radiosurgery using the Leksell Gamma Knife Perfexion unit in the management of patients with 10 or more brain metastases. Clinical article. J Neurosurg 117:237-245, 2012

11. Gray RJ: A class of K-sample tests for comparing the cumulative incidence of a competing risk. Ann Stat 16:1141-1154, 1988

12. Hanssens P, Karlsson B, Yeo TT, Chou N, Beute G: Detection of brain micrometastases by high-resolution stereotactic magnetic resonance imaging and its impact on the timing of and risk for distant recurrences. Clinical article. J Neurosurg 115:499-504, 2011

13. Hayashi M, Yamamoto M, Nishimura C, Satoh H: Do recent advances in MR technologies contribute to better gamma knife radiosurgery treatment results for brain metastases? Neuroradiol J 20:481-490, 2007

14. Hunter GK, Suh JH, Reuther AM, Vogelbaum MA, Barnett $\mathrm{GH}$, Angelov L, et al: Treatment of five or more brain metastases with stereotactic radiosurgery. Int J Radiat Oncol Biol Phys 83:1394-1398, 2012

15. Kano H, Kondziolka D, Lobato-Polo J, Zorro O, Flickinger JC, Lunsford LD: T1/T2 matching to differentiate tumor growth from radiation effects after stereotactic radiosurgery. Neurosurgery 66:486-492, 2010

16. Kaplan EL, Meier P: Nonparametric estimation from incomplete observations. J Am Stat Assoc 53:457-481, 1958

17. Karlsson B, Hanssens P, Wolff R, Söderman M, Lindquist C,
Beute G: Thirty years' experience with Gamma Knife surgery for metastases to the brain. Clinical article. J Neurosurg 111: 449-457, 2009

18. Karnofsky DA, Buechenal JH: The clinical evaluation of chemotherapeutic agents in cancer, in MacLeod CM (ed): Evaluation of Chemotherapeutic Agent. New York: Columbia University Press, 1949, pp 191-205

19. Kim CH, Im YS, Nam DH, Park K, Kim JH, Lee JI: Gamma knife radiosurgery for ten or more brain metastases. J Korean Neurosurg Soc 44:358-363, 2008

20. Knisely JPS, Yamamoto M, Gross CP, Castrucci WA, Jokura H, Chiang VLS: Radiosurgery alone for 5 or more brain metastases: expert opinion survey. Clinical article. J Neurosurg 113 Suppl:84-89, 2010

21. Linskey ME, Andrews DW, Asher AL, Burri SH, Kondziolka $\mathrm{D}$, Robinson PD, et al: The role of stereotactic radiosurgery in the management of patients with newly diagnosed brain metastases: a systematic review and evidence-based clinical practice guideline. J Neurooncol 96:45-68, 2010

22. Nariai T, Tanaka Y, Wakimoto H, Aoyagi M, Tamaki M, Ishiwata K, et al: Usefulness of L-[methyl-11C] methioninepositron emission tomography as a biological monitoring tool in the treatment of glioma. J Neurosurg 103:498-507, 2005

23. Matsuo M, Miwa K, Shinoda J, Kako N, Nishibori H, Sakurai $\mathrm{K}$, et al: Target definition by C11-methionine-PET for the radiotherapy of brain metastases. Int J Radiat Oncol Biol Phys 74:714-722, 2009

24. Radiation Therapy Oncology Group: Cooperative Group Common Toxicity Criteria. (http://www.rtog.org/Research Associates/AdverseEventReporting/CooperativeGroup CommonToxicityCriteria.aspx) [Accessed March 18, 2013]

25. Rosenbaum PR, Rubin DB: The central role of the propensity score in observational studies for causal effects. Biometrika 70:41-55, 1983

26. Satagopan JM, Ben-Porat L, Berwick M, Robson M, Kutler $\mathrm{D}$, Auerbach AD: A note on competing risks in survival data analysis. Br J Cancer 91:1229-1235, 2004

27. Serizawa T, Hirai T, Nagano O, Higuchi Y, Matsuda S, Ono $\mathrm{J}$, et al: Gamma knife surgery for 1-10 brain metastases without prophylactic whole-brain radiation therapy: analysis of cases meeting the Japanese prospective multi-institute study (JLGK0901) inclusion criteria. J Neurooncol 98:163-167, 2010

28. Serizawa T, Yamamoto M, Sato Y, Higuchi Y, Nagano O, Kawabe T, et al: Gamma Knife surgery as sole treatment for multiple brain metastases: 2 -center retrospective review of 1508 cases meeting the inclusion criteria of the JLGK0901 multi-institutional prospective study. Clinical article. J Neurosurg 113 Suppl:48-52, 2010

29. Sheehan J, Schlesinger D: Editorial. Ten brain metastases. J Neurosurg 117:234-236, 2012

30. Shuto T, Fujino H, Inomori S, Nagano H: Repeated gamma knife radiosurgery for multiple metastatic brain tumours. Acta Neurochir (Wien) 146:989-993, 2004

31. Sperduto PW, Sneed PK, Roberge D, Shanley R, Luo X, Luo $\mathrm{X}$, et al: Editorial. In regard to Yamamoto et al. Int J Radiat Oncol Biol 84:875-877, 2012

32. Suzuki S, Omagari J, Nishio S, Nishiye E, Fukui M: Gamma knife radiosurgery for simultaneous multiple metastatic brain tumors. J Neurosurg 93 (3 Suppl 3):30-31, 2000

33. Tsao MN, Rades D, Wirth A, Lo SS, Danielson BL, Gasper LE, et al: Radiotherapeutic and surgical management for newly diagnosed brain metastasis(es): an American Society for Radiation Oncology evidence-based guideline. Practic Radiat Oncol 2:210-225, 2012

34. Tsuyuguchi N, Sunada I, Iwai Y, Yamanaka K, Tanaka K, Takami T, et al: Methionine positron emission tomography of recurrent metastatic brain tumor and radiation necrosis after 
stereotactic radiosurgery: is a differential diagnosis possible? J Neurosurg 98:1056-1064, 2003

35. Yamamoto $\mathbf{M}$, Ide $M$, Jimbo $M$, Aiba $M$, Ito $M$, Hirai $H$, et al: Gamma knife radiosurgery with numerous target points for intracranially disseminated metastases: early experience in three patients and experimental analysis of whole brain irradiation doses, in Kondziolka D (ed): Radiosurgery 1997. Basel, Switzerland: Karger, 1998, Vol 2, pp 94-109

36. Yamamoto M, Ide M, Nishio Si, Urakawa Y: Gamma Knife radiosurgery for numerous brain metastases: is this a safe treatment? Int J Radiat Oncol Biol Phys 53:1279-1283, 2002

37. Yamamoto M, Kawabe T, Barfod BE: How many metastases can be treated with radiosurgery? Prog Neurol Surg 25:261272, 2012

38. Yamamoto M, Kawabe T, Higuchi Y, Sato Y, Narai T, Barfod BE, et al: Delayed complications in patients surviving at least three years after stereotactic radiosurgery for brain metastases. Int J Radiat Oncol Biol Phys 85:53-60, 2013

39. Yamamoto M, Sato Y, Serizawa T, Kawabe T, Higuchi Y, Nagano O, et al: Subclassification of recursive partitioning analysis Class II patients with brain metastases treated radiosurgically. Int J Radiat Oncol Biol Phys 83:1399-1405, 2012

Manuscript submitted October 4, 2012.

Accepted March 14, 2013.

Please include this information when citing this paper: published online April 19, 2013; DOI: 10.3171/2013.3.JNS121900.

Address correspondence to: Masaaki Yamamoto, M.D., Katsuta Hospital Mito GammaHouse, 5125-2 Nakane, Hitachi-naka, Ibaraki 312-0011, Japan.email: BCD06275@nifty.com. 